\section{Food-Borne Parasitic Zoonoses: Fish and Plant- Borne Parasites (World Class Parasites)}

\author{
K. Darwin Murrell and \\ Bernard Fried, editors \\ Springer, New York, New York, \\ USA, 2007 \\ ISBN-10: 0387713573 \\ ISBN-13: 978-0-387-71357-1 \\ Pages: 686; Price: US \$169
}

With $>4,000$ biomedical journals currently available, and more being added seemingly every month, one of the most overwhelming and daunting tasks for any health professional is to stay current with the literature. A welcomed lifeline has been the World Class Parasites series of publications that have made this challenge much easier to manage. The latest text (Volume 11), Food-Borne Parasitic Zoonoses: Fish and Plant-Borne Parasites, is a superb addition to this series. Murrell and Fried have brought together a stellar group of contributors in a comprehensive and up-to-date review of the helminthic zoonoses that are transmitted through fish and plant consumption. Given that the distribution and effect of these zoonoses remain woefully underappreciated, they are deserving of the justifiable attention brought by this book.

The first 9 chapters provide a thorough discussion of intestinal, liver, and lung flukes; fish-borne tapeworms; and tissue nematodes. Two superb chapters on immunologic aspects and molecular epidemiology of these parasites complete the text. There is much to extol in this volume. In an era of justifiable focus on molecular biology and genetics, it is heartening to see the broad-based and integrated approach taken by the editors and collaborators. This approach successfully accomplishes the stated objec- tive of the editors to "... celebrate the diversity of approach that comprises modern parasitological research." The chapters are clearly written and well organized with a balance of both classic, historic articles as well as important recent work.

The editors and authors have obviously made a priority of ensuring that the material is widely accessible to scientists and health professionals in diverse disciplines. They have presented detailed information on the biology, life cycles, natural history, diagnosis, treatment, epidemiology, and control of the agents or infections discussed. Current diagnostic techniques are thoroughly addressed. Insightful discussion is provided at the end of each chapter on current gaps in knowledge and research needs that offers a coherent roadmap for future studies. The chapter on intestinal capillariasis by Cross and Belizario is particularly noteworthy. Abundant images of exceptional quality are included and this adds substantially to the text. More color images would have been of value, but they may have made the text prohibitively expensive. Some chapters could have had additional discussion of epidemiologic aspects and a few typos can be found, but these do not diminish the value of this outstanding work.

Food-Borne Parasitic Zoonoses: Fish and Plant-Borne Parasites is a text of exceptional quality that should have broad appeal and utility for health professionals, researchers, medical and public health students, and policy makers. Given the increasing interest in neglected tropical diseases, this book is a timely and commendable work that will promote efforts to reduce the effects of these parasitic agents.

\section{Frank J. Sorvillo}

Author affiliation: University of California, Los Angeles, California, USA

DOI: 10.3201/eid1409.080495
Address for correspondence: Frank J. Sorvillo, Department of Epidemiology, School of Public Health, University of California, Los Angeles, CA 90095, USA; email: fsorvill@ucla.edu

\section{Epidemiologic Principles and Food Safety}

\section{Tamar Lasky, editor}

Oxford University Press, New York, New York, USA, 2007

ISBN-10: 0-19-517263-9

ISBN-13: 978-0-19-517263-8

Pages: 272; Price: US \$49.95

This text is an ambitious overview of the wide field of public health and food safety. Topics include foodborne pathogens, public health surveillance, basic epidemiologic principles, study design and outbreak management, food production, and food safety regulation. A substantial amount of attention has focused recently on the complexity of the food safety system in the United States, which includes a huge number of agencies, sometimes overlapping programs, databases and legal authority, and the inevitable consequence of inefficient and confusing communication. In light of these circumstances, this text provides a pertinent overview of the system that will be useful even to those very familiar with a particular aspect of the food safety continuum.

The descriptions of existing surveillance systems, sources of food consumption data, the federal regulatory system approach to risk assessment, food production and controls, and the regulatory environment are particularly clear, useful, and timely descriptions of sometimes vexingly complex systems. Clearly, such a breadth of topics cannot be addressed 
in great detail in a single text, but many of the subjects are seldom summarized so cogently, and readers interested in exploring particular topics will find this text provides a nice foundation for delving more deeply into the literature. This book provides something for everyone-an excellent overview for students, epidemiologists, regulators, academicians, industry representatives, and others interested in a broad survey of the field.

\section{Timothy Jones}

Author affiliation: Tennessee Department of Health, Nashville, Tennessee, USA
DOI: 10.3201/eid1409.080585

Address for correspondence: Timothy Jones, Tennessee Department of Health, Communicable and Environmental Disease Services, Cordell Hull Bldg, 425 5th Ave N, 1st Floor, Nashville, TN 37243, USA; email: tim.f.jones@ state.tn.us
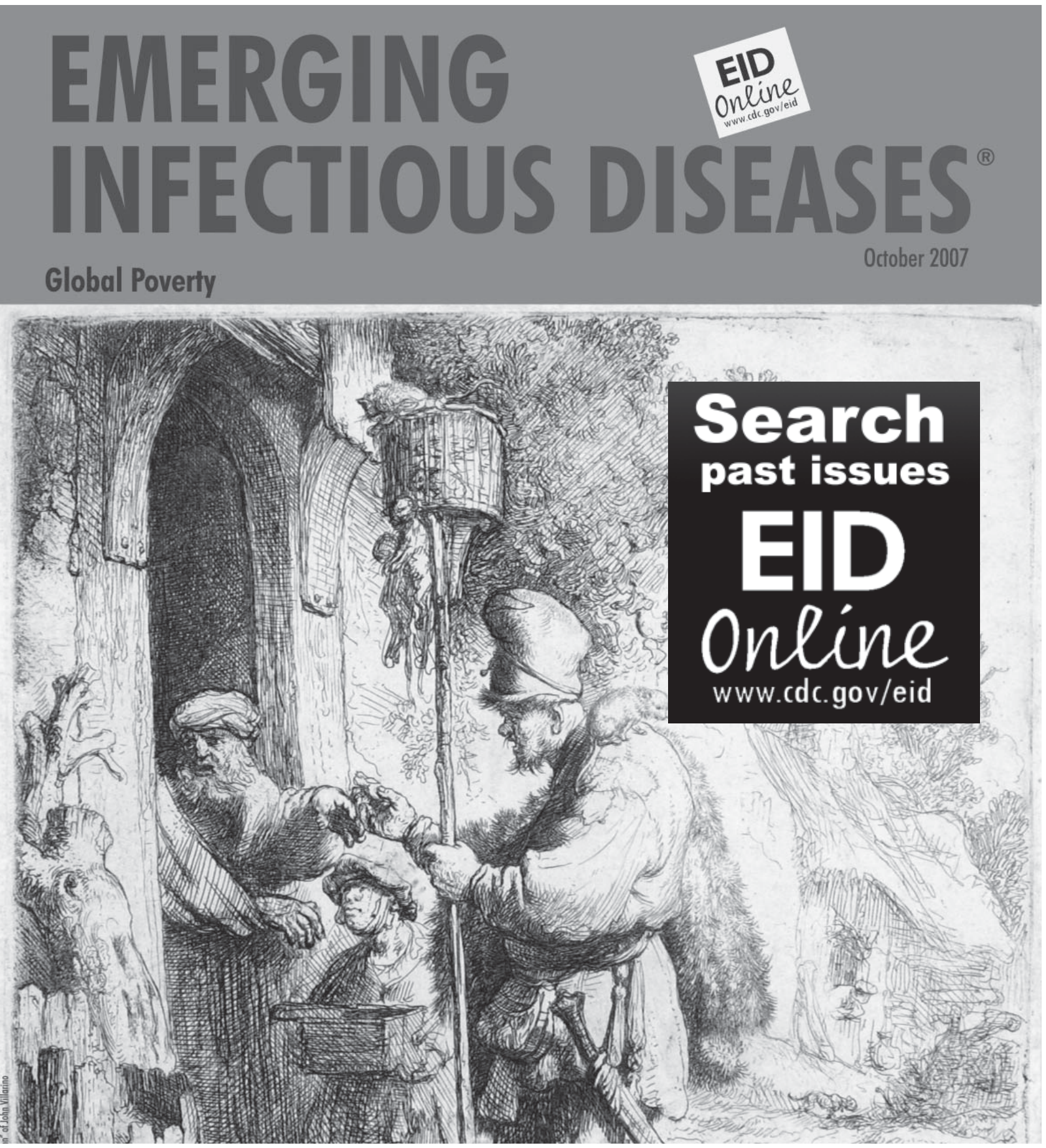